12th.-Saw her this morning in consultation with my friend Dr. Bush; found her very much as on the day previous; continued the sane treatment, adding the cornpound tinct. of catechu to be applied to the nose, and given internally.

13th.- Still bleeding at the nose, though much less profusely; continued the creosote, as it seemed to be the only application which was of any service; symptoms much the same as yesterday; hysteria still continues; treatment the same, only syrup morphine added to control the nervous irritability.

14th.- This morning found my patient relieved from hemorrbage from the nose ; pulse small and frequent; skin moist ; extremities cool; exceedingly feeble, and easily excited to crying or laughter; mouth sore, cheeks swollen, notwithstanding the bowels have been opened every day with mild cathartics. This day the catamenia came on, and at times was somewhat profuse, producing the greatest alarm, but all persuasions of her mother or myself were unavailing to induce ber to suffer any local application to be made. Continued general treatment; added tinct. myrrh in drachm doses. She now began to improve slowly, and was furnished with such medicines from the shop as her condition required from time to time.

December 3.-Was called to my patient again; on arriving 1 found ber laboring under uterine hemorrhage in the strict sense of the word. This, of course, produced much alarm. I explained the nature of the case, and assured her that there was no danger, as it was of very frequent occurrence, though not in unmarried women. It readily yielded to the usual treatment; since which time she has recovered her ustral health and spirits.

I might mention, in conclusion, that this young lady belongs to one of the most respectable fanilies in the farming community, who never knew want, or participated in luxurious indulgences. I present the case as it occurred, without comment. How far the powerful agents she was using had any share in producing the state of things above recited, I shall not attempt to determine. The pathology of purpura is still involved in great obscurity. The multiplication of cases must ultimately clear it up. In that view, I trust the one which I have given may be found to be of some value.-Western Journal of Medicine and Surgery.

\title{
UTILITY OF A KNOWLEDGE OF THE TEMPERAMENTS.
}

By F. Robinson, Esqq, Surgeon, Hammersmith.

THAT different temperaments appear in a certain degree to influence the performance of various actions differing in their nature, is a fact that seems to have been tolerably well established by acute observers of human nature. It is only, however, at a comparatively late period, that the connection existing between the various expressions of the countenance and the temper has been at all definitely pointed out, and the fact that the former are in a greater or less degree symbolical of the latter established 
beyond doubt. Phrenologists are evidently aware of the utility of this circumstance, in giving additional strength to their doctrines, and generally call it to their aid in forming an opinion of the cranial development of an individual. But the importance of a knowledge of the temperaments, their various characteristics and influences in medicine, appeared to meet with little of the attention it deserved, and to have passed almost wholly unnoticed by writers, till the time of Lavater. Even that writer, in his Essay on Physiognomy, only mentions superficially, and in somewhat vague terms, the benefit that might be derived from a study of this branch in connection with disease.

I shall endeavor to point out briefly some of the principal advantages accruing from its study, which appear to me worthy of attention, as tending to elucidate the intricacies of disease : but before doing so, may be petmitted to state that I do not lay claim to originality in some of the following remarks, which have been, and probably are being, daily observed by physicians in extensive practice, my object being to call some notice to what, from the little mention made of it by writers of the present day, appears to be considered an unimportant, if not decidedly useless study. That the fathers of the profession, when it was yet in its infancy, deemed it worthy of the closest attention at a time when their knowledge of disease was very limited (comparatively), their means of alleviating it few and simple, and of those few, many depending, not improbably, for their success upon their influence on the patient's own imagination, is extremely likely, and is, in fact, partly confirmed in their own writings. I would observe, then,

1. That certain remedies possess different degrees of effect in their action on individuals of different temperaments. This may frequently be noticed by physicians in large practice, as a medicine which will act almost as a specific in controlling the progress of disease in an individual of one temperament, will be found inert, if not positively injurious, in a like disease and same stage in another. This might, in an individual case, be attributed to that inexplicable condition termed idiosyncrasy ; but whoever has opportunities of trying one remedy for a fixed period on persons of all temperaments (their complaints, of course, being of the same nature and duration), and then carefully analyzes the results, will in all probability be convinced to the contrary. Undoubtedly, be would meet with occasional instances where a remedy possessing considerable power in these cases-in the majority of them-fails in an individual one, without any apparent cause to account for it ; and if, in conjunction with this, a minimum dose of the medicine produces the effect of a maximum, or entirely different symptoms, this would appear to me to merit the appellation of idiosyncrasy (as far as our knowledge of the term permits us to employ it), provided, of course, that nothing abnormal is detected in the individual that could at all affect the action of the remedy.

It must be confessed, also, that there are numeroùs diseases, incident to individuals of all temperaments, in which no distinction can be drawn in the action of medicines, as they may one or all fail ar succeed withaut 
our being able to explain "why." Are we not in the habit of trying one, and, in the event of failure, commencing with another, approved remedy for a disease, without stopping to consider the cause of that remedy failing? It being thus proved that a remedy possesses a greater degree of efficacy in a disease occurring in a person of one temperament than another, how are we to account for this circumstance? Provided no abnormal condition exists, it appears to me that, as far as our present knowledge extends, we can only attribute this peculiarity to one cause, and that, necessarily, an obscure one, viz., that the organization of the nervous system modifies, in some (as yet hidden) manner, the action of the remedy, or its absorption. That this modus operandi exists in the healthy human frame, when subjected to the action of one class of stimuli, viz., alcoholic, is proved, I think, beyond doubt.

Dr. Macnish, in his "Anatomy of Drunkenness," has shown the very opposite effects produced by intoxicating liquors on individuals of different organism, as well as the various degrees of rapidity in producing its action on individuals equally unaccustomed to its stimulus. Judging from these facts, it does not appear improbable that the action of some medicines, as, for instance, diffusible stimuli, is materially influenced by the temperaments in health, and also disease. Let us suppose that an individual takes a quantity of some narcotic poison, opium-for example, whose action is primarily to stimulate-and suffers it to remain for a period that, in many persons, would prove fatal. Nevertheless, the proper remedial measures being adopted, he recovers, in some instances, and this is invariably attributed by the patient's friends and medical attendant to the individual's constitution, previous good health, \&c. ; or some other cause known to affect the action of the poison, as the amount of food in the viscus, without considering the possibility of one person's frame being more susceptible to the action of the poison than another's. It is not, as far as I am aware, in opposition to any known physiological principles, to suppose that opium would be capable of producing quicker and more deadly effects on a person of thin, spare habit (though of sound health), and highly nervous, bilious temperament, than a large robust individual of lymphatic sanguineous temperament. A case of accidental poisoning that occurred to me to treat lately, strengthened this opinion.

2. That certain classes of diseases are particularly liable to originate in persons of certain temperaments.

This is a circumstance that cannot well escape the observation of an attentive practitioner, and in large hospitals especially. I may briefly mention, as examples, the occurrence of acute rheunatism, and, consequently, often of pericarditis, in individuals in whose temperaments the sanguine predominates. Anasarca and ascites, dependent on renal disease, in persons of lymphatic, or lymphatic sanguineous temperaments, and ascites, dependent on hepatic disease, in those in which the bilious predominates. A careful comparison of cases convinced ine of the truth of this theory in a general point of view ; and my observations not being confined to individuals of the same trade and habits, but all the numerous classes of artizans met with in a large manufacturing and com- 
mercial district, they cannot be said to be derived from, or rendered applicable to, one class of mechanics only. A greater tendency also appeared to me to exist, to the generation of organic disease, perhaps especially that of the liver, in individuals of highly bilious nervous temperament; and this bypothesis would appear to be partly confirmed by the observation of a recent writer, who mentions the comparative frequency of carcinoma of the uterus (in a number of cases treated by him) in females of highly bilious temperament.

With regard to the utility of a knowledge of the temperaments in surgical diseases and accidents, the principal benefit that I conceive to be derived is, in many instances, to render the diagnosis and prognosis more clear and certain, and thus afford material assistance to him in some cases.

It has been my endeavor, in the foregoing remarks, to treat as concisely as possible some of the leading points connected with a subject that admits of very extensive research, even if one not capable of affording material assistance to the physician in the diagnosis and treatment of disease, at least one that might tend to elucidate some of those as yet hidden phenomena which we meet with in practice; and my object, in giving publication to these brief and imperfect remarks is-through the medium of the Lancet -if possible, to draw attention to the subject. It is now a year since most of the foregoing notes were made, at a time when enjoying considerable opportunities for so doing, and my subsequent experience has tended to confirm the opinions I entertained then.-London Lancet.

\section{CASE OF INVERSION OF THE UTERUS.}

By घ. Fisher, M.D., of Waynesville, Ohio.

$\mathrm{ON}_{\mathrm{N}}$ the $26 \mathrm{th}$ of September, 1835, at 10 o'clock, A. M., I was called to Mis. D-, in labor. She was about 35 years of age, and had given birth to several children-said that two weeks previously, in attending the funeral of a relative, she rode several uniles in a farm-waggon over rough ronds, which excited pain in the loins and hips, and weakness of the inferior extremities, attended with difficulty of locomotion; all of which continued up to the morning of the $26 \mathrm{th}$.

From the day of the funeral she had felt no motion of the fotus, and, to use her own language, she was " eight months gone in pregnancy." The pains were slight, irregular and transient. Upon examination I found the pelvis unusually large, the os uteri well dilated, and the membranes protruding. The temperature of the skin was natural, pulse regular, and bowels open.

As I conceived there was but little to fear, I deemed it prudent to give nature time to effect her purpose. Ten grains of pulris Doveri were administered, which procured an hour's repose. The pains then returned, but continued feeble an hour longer without any appreciable change, when suddenly a violent throe thrust foetus, placenta, and body of the uterus beyond the labia exteria. 